\title{
mesure des propriétés dynamiques des sols
}

\author{
measurement of dynamic soil characteristics
}

M.P. LUONG

Maître de recherche C.N.R.S. UA 317

Laboratoire de Mécanique des Solides*

E.P. - E.N.S.M.P. - E.N.P.C.

\section{Résumé}

Le texte présente successivement les différentes techniques de mesure - en laboratoire et in situ - des principales caractéristiques dynamiques des sols modules de déformation et coefficients d'amortissement — sur une large gamme de déformation intéressant les calculs de réponse sismique des ouvrages de génie civil.

\section{Abstract}

The paper reviews the different measurement techniques - in laboratory and in field - of deformation moduli and damping ratio, over a wide range of deformation amplitude being of interest for earthquake resistant design of engineering structures. 


\section{INTRODUCTION}

Des progrès considérables ont été réalisés en dynamique des sols au cours de ces dernières décades aussi bien en investigation de laboratoire qu'en expérimentation sur le site. Ils ont permis une meilleure connaissance du comportement des sols soumis aux sollicitations cycliques, vibratoires, transitoires et dynamiques. lls ont surtout offert des techniques fiables de mesure des paramètres physiques indispensables aux concepteurs d'ouvrages en cas de calcul de réponse dynamique.

En effet, les problèmes de l'ingéniérie moderne couvrent une vaste gamme de situations : depuis les très petits mouvements admis pour les fondations des installations pour laser ou des télescopes, jusqu'aux mouvements destructeurs des séismes ou des explosions nucléaires. Cette principale difficulté a stimulé le développement de nombreuses techniques de laboratoire et d'innombrables procédures expérimentales en place pour évaluer d'une manière réaliste les propriétés mécaniques du sol, si possible utilisables sur plusieurs décades de déformation relative. Les principales caractéristiques à prendre en compte par les méthodes numériques de calcul de réponse dynamique des structures sont :

1. Caractéristiques de déformation : module dynamique, module d'Young $\mathrm{E}$, module de cisaillement $\mathrm{G}$, module volumique $\mathrm{K}$, raideur contenue, coefficient de Poisson $x, \ldots$.

ii. Caractéristiques de dissipation d'énergie : amortissement, dispersion, atténuation, ....

iii. Caractéristiques de liquéfaction : nombre de cycles conduisant à la liquéfaction totale, coefficient de cisaillement cyclique, réponse en déformation cyclique et en pression interstitielle, ...

iv. Caractéristiques rhéologiques : effets des vitesses de déformation ou de contrainte.

Certains paramètres sont mieux évalués ou étudiés in situ, d'autres en laboratoire, et un certain nombre peuvent être mesurés à la fois en laboratoire et sur le site.

\section{NATURE DU COMPORTEMENT CONTRAINTE-DEFORMATION DES SOLS}

La dynamique des sols se réfère généralement au comportement élastique ou pseudoélastique dans le domaine des faibles déformations alors que la statique ne s'intéresse pratiquement qu'aux caractéristiques asymptotiques à la rupture dans le cas des grandes déformations. Il est intéressant de rappeler le problème du contact de deux grains sphériques élastiques pressés l'un vers l'autre (MINDLIN et DERESIEWICZ, 1953) pour mieux préciser la nature du comportement d'un assemblage de particules solides constituant le sol.

Au point de contact, la réaction intergranulaire est supposée obéir aux lois de frottement solide : le rapport de la composante tangentielle à la composante normale est inférieur au coefficient de frottement $\mathrm{f}$. Les déformations des grains sont supposées élastiques et il n'y a aucune rupture : les forces intergranulaires sont peu élevées et la courbure des surfaces en contact est faible.

Dans le cas très simple de deux sphères identiques de rayon $\mathrm{R}$ comprimées statiquement par une force $\mathrm{N}$ dirigée suivant la ligne des centres, normale au plan tangent commun, la théorie de contact de Hertz prédit un contact plan circulaire de rayon :

$$
a=\left[3\left(1-v^{2}\right) N R / 4 E\right]^{1 / 3}
$$

E et $v$ sont le module d'Young et le coefficient de Poisson du matériau constituant les sphères.

Sur le cercle de contact, la contrainte est uniquement normale (en première approximation) et sa valeur est :

$$
\sigma=3 N\left(a^{2}-p^{2}\right)^{1 / 2} / 2 \pi a^{3}
$$

où $\rho$ représente la distance du point à la ligne des centres.

Une force additionnelle T est supposée agir dans le plan de contact avec une intensité croissant progressivement de zéro à une certaine valeur.

A cause de la symétrie, la distribution de pression normale reste inchangée. Si aucun glissement ne se produit sur cette surface de contact, la contrainte de cisaillement $\tau$ a pour valeur :

$$
\tau=\mathrm{T} / 2 \pi \mathrm{a}\left(\mathrm{a}^{2}-\rho^{2}\right)^{1 / 2}
$$

La contrainte de cisaillement $\tau$ est infinie sur les bords. Ce qui est impossible si l'on admet la validité de la loi de frottement de COULOMB: $|\tau| \leqslant f(\sigma)$.

Un glissement apparaît sur les bords du cercle de contact et se propage vers l'intérieur formant un anneau de glissement de rayons a et $c$ (fig. 1).

$$
c=a[1-(T / f N)]^{1 / 3}
$$

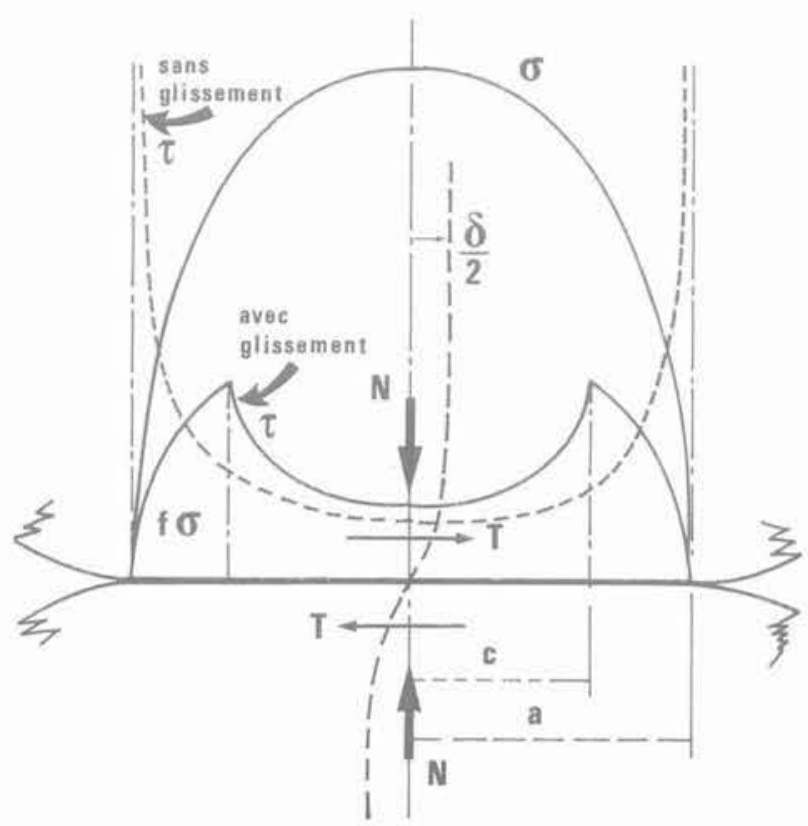

Fig. 1. - Contraintes au contact de Hertz. 
On se rend ainsi compte que le phénomène est affecté d'une certaine irréversibilité due au glissement de la portion annulaire de la plage de contact.

La zone de glissement augmente avec $\mathrm{T}$ pour recouvrir toute la surface de contact lorsque $\mathrm{T}=\mathrm{fN}$.

Après avoir atteint une valeur $\mathrm{T}^{*}$ avec $0<\mathrm{T}^{*}<\mathrm{fN}$, si la force tangentielle $T$ est réduite, il faut à nouveau supposer qu'un glissement se produit dans la direction opposée au glissement initial (croissant de 0 à T**). Un anneau de contre-glissement est formé sur les bords et se propage radialement vers l'intérieur au fur et à mesure que $T$ diminue. Son rayon intérieur est :

$$
\mathrm{b}=\mathrm{a}\left[1-\left(\mathrm{T}^{*}-\mathrm{T}\right) / 2 \mathrm{fN}\right]^{1 / 3}
$$

avec $c \leqslant b \leqslant a$ tant que $-\mathrm{T}^{*}<\mathrm{T}<+\mathrm{T}^{*}$.

Le caractère inélastique lors de la décharge apparaît évident car l'anneau de contre-glissement persiste lorsque la force tangentielle $\mathrm{T}$ s'annule (fig. 2).
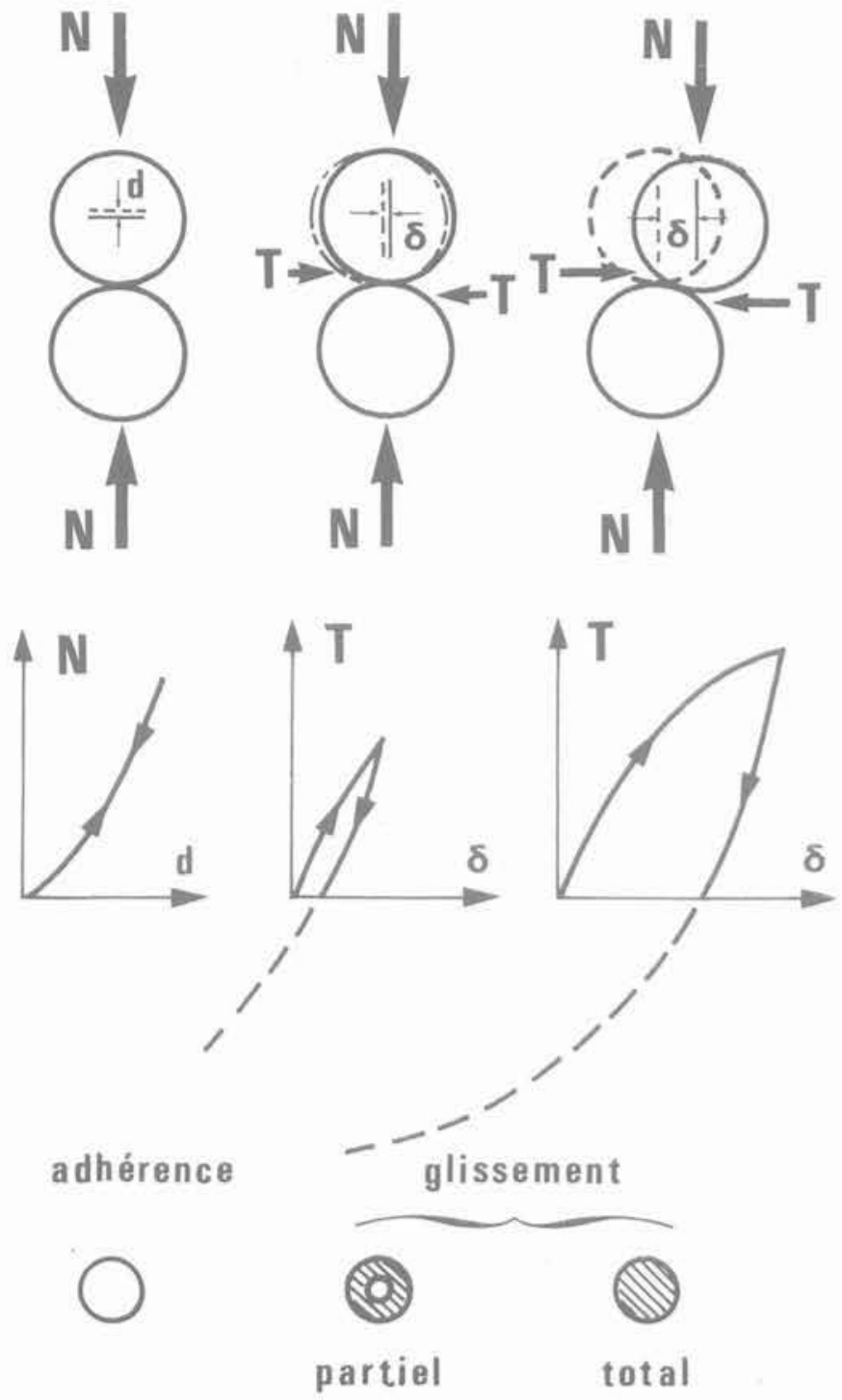

Fig. 2. - Glissements au contact de Hertz.
Si l'on fait osciller la force tangentielle entre $+T^{*}$ et $-T^{*}$, la courbe effort-déformation devient un cycle fermé. L'aire du cycle représente le travail dissipé sous forme de frottement. Sa valeur est :

$$
\mathrm{W}=(2-\nu) \mathrm{T}^{*} 3 / 36 \mu \mathrm{afN}
$$

où $\mu$ est le module de glissement de L.AME.

Ainsi on peut constater que les réactions tangentielles (mécanisme de distorsion), contrairement aux réactions normales (mécanisme de consolidation), donnent lieu à des déformations inélastiques et à des dissipations d'énergie. A faible amplitude de la force tangentielle $\left(T^{*}<\mathrm{fN}\right)$, l'énergie dissipée résulte des déformations plastiques sur une petite portion de la surface de contact. A plus forte amplitude $\mathrm{T}^{*}>\mathrm{fN}$, l'effet du glissement de COULOMB prédomine par une grande dissipation d'énergie en chaleur.

Le schéma de MINDLIN montre que la réversibilité des déformations n'implique pas l'inexistence de dissipation d'énergie, qui constitue la source du phénomène d'amortissement.

Une part de la déformation du sol est due à la déformation élastique des particules solides. Elle est d'autant plus significative que la sollicitation de cisaillement au point de contact est petite. C'est le cas des petits mouvements dans le sol. Au contraire, lorsque l'amplitude de la déformation totale devient importante, la part élastique peut devenir très petite et, de ce fait, souvent masquée par la déformation irréversible résultant du glissement, du ré-arrangement avec variation du nombre de contacts intergranulaires et de l'écrasement éventuel des particules. Examinons successivement ces différents cas.

\section{PETITS MOUVEMENTS DANS LE SOL}

Les petits mouvements au voisinage d'un état d'équilibre quasi naturel sont régis par les équations suivantes:

- équation de continuité,

- équations dynamiques,

- relations de comportement,

- équation thermique.

L'ensemble définit une propagation complexe des ondes à travers le milieu car l'intervention de la conduction, phénomène irréversible, tout comme la viscosité entraîne :

- une diminution progressive de la fonction $U-T S$ $+W+C(U$ énergie interne, $\mathrm{S}$ entropie, $\mathrm{W}$ énergie potentielle des forces extérieures et $\mathrm{C}$ énergie cinétique) ;

- un amortissement des ondes sinusoidales ou ondes de phase. Leur célérité est fonction de leur fréquence à cause de la dispersion.

Dans l'hypothèse de l'adiabaticité, on constate que : 
$1^{\circ}$ la dilatation cubique $\theta$ se propage par ondes longitudinales de dilatation de célérité :

$$
V_{L}=\sqrt{\frac{\lambda+2 \mu}{\rho}}
$$

$2^{\circ}$ la rotation se propage par ondes transversales de rotation de célérité :

$$
V_{\mathrm{s}}=\sqrt{\frac{\mu}{\rho}}
$$

Ces mouvements satisfont séparément à l'équation indéfinie :

$$
\left(\lambda^{\prime}+\mu\right) \operatorname{grad} \operatorname{div} \vec{\xi}+\mu \Delta \vec{\xi}-\rho \frac{\partial^{2} \vec{\xi}}{\partial t^{2}}=0
$$

mais non aux conditions à la surface dans le cas d'un milieu élastique limité.

La réflexion des ondes à la surface complique les phénomènes :

- une onde de cisaillement polarisée dans le plan d'incidence i donne naissance à une onde de cisaillement et une onde de dilatation réfléchie sous un angle $r$ tel que $\sin r=\frac{V_{L}}{V_{S}} \sin i$ lorsque $\sin i<\frac{V_{S}}{V_{L}}$;

- dans le cas où $\sin i>\frac{V_{S}}{V_{L}}$, l'onde de dilatation est remplacée par une onde de surface qui se propage parallèlement à la surface, et reste localisée au voisinage de celle-ci. Cette onde de RAYLEIGH de célérité un peu inférieure à $V_{S}$ se disperse dans deux dimensions tandis que les deux autres se propagent dans trois dimensions.

\section{PROPAGATION D'ONDES}

La célérité des ondes ultrasonores est analysée le plus souvent en laboratoire, par la méthode de transparence à l'aide des céramiques ferro-électriques comme émetteur et récepteur. La technique utilisée (NAUROY, 1975) est dite première arrivée d'impulsion $(0,2$ à $2 \mathrm{MHz}$ ). On mesure le temps de transit d'un train d'ondes longitudinales ou transversales à travers une épaisseur connue de sol soumis à une compression œdométrique ou triaxiale cylindrique (fig. 3).

Les vitesses de propagation des ondes longitudinales et transversales $V_{L}$ et $V_{S}$ varient en fonction de la porosité et de la contrainte appliquée (fig. 4). On en déduit les modules dynamiques d'YOUNG $\mathrm{E}$ et de cisaillement $\mathrm{G}=\mu$. On peut constater que par sa morphologie, un sol sableux joue le rôle d'un filtre de fréquence passe-bas. La granulométrie et la porosité régissent latténuation des ondes élastiques. Le matériau sec se comporte comme un solide élastique continu avec une onde longitudinale et une onde transversale. Le matériau saturé d'eau se présente comme un solide poreux de Biot. Les paramètres mécaniques sont fonction de la porosité et de la contrainte appliquée.

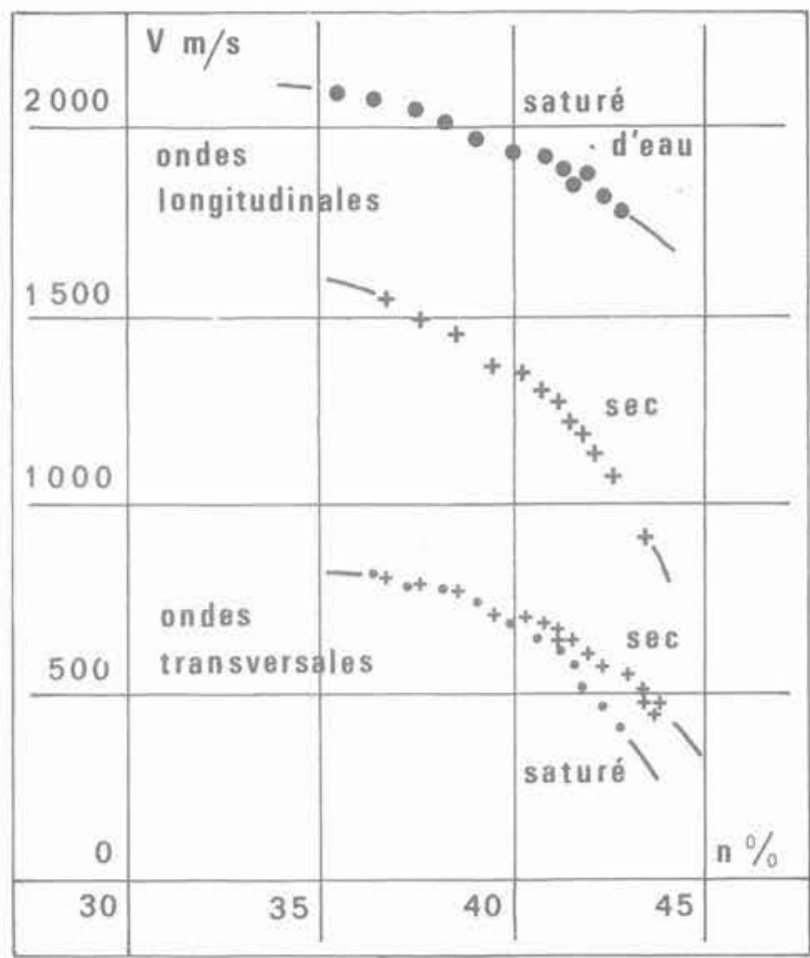

Fig. 4. - Célérité des ondes acoustiques en fonction de la porosité de l'échantillon Texsol-Fontainebleau.

In situ, la propagation d'ondes ultrasonores est analysée par des essais sismiques en forage ou de surface.

La distinction entre essais en forage entre trou (crosshole), en descendant (down-hole), en remontant (up-hole), dans un seul trou (in-hole) ou en fond de trou (bottom-hole) est faite d'après les positions relatives de la source et des récepteurs, suivant le volume testé, ce qui conditionne la trajectoire des ondes sismiques.

Les essais de surface, profitant de l'avantage de la rapidité d'exécution, d'une étendue plus grande et d'un coût relativement modéré, analysent les phénomènes de réfraction-réflexion, ainsi que les ondes de RAYLEIGH stationnaires ou en dispersion lors d'une source impulsionnelle.

Les modules d'YOUNG E, de cisaillement $G$ et le coefficient de Poisson v sont déduites des vitesses de propagation longitudinale et transversale par :

$$
\begin{aligned}
E=2 \rho V_{\xi}(1+\nu) & =\rho V_{L}^{2} \frac{(1+\nu)(1-2 \nu)}{1-\nu} \\
G & =\rho V_{\xi}
\end{aligned}
$$

Le niveau de déformation est estimé par :

$\mathcal{E}=\dot{\mathrm{u}}_{\mathrm{L} / \mathrm{V}_{\mathrm{L}}} \quad$ dans le cas des ondes longitudinales et $\gamma=\dot{\mathrm{u}}_{\mathrm{s} / \mathrm{v}_{\mathrm{S}}}$ lorsqu'il s'agit d'ondes de cisaillement.

$\mathcal{E}, \dot{\mathrm{u}}_{\mathrm{L}}$ et $\gamma$, $\dot{\mathrm{u}}_{\mathrm{S}}$ sont respectivement la déformation et la vitesse particulaire des ondes longitudinales et transversales. 
L'ordre de grandeur de l'amplitude des déformations est inférieur à $10^{-5}$.

Le coefficient d'amortissement est déduit des essais de propagation d'ondes par différentes méthodes :

- Rapport spectral (spectral ratio) déterminé par la décroissance des amplitudes des ondes en s'éloignant de la source. Le taux de la variation de la pente spectrale donnée par des mesures à différentes distances de la source donne une constante d'atténuation « a » reliée
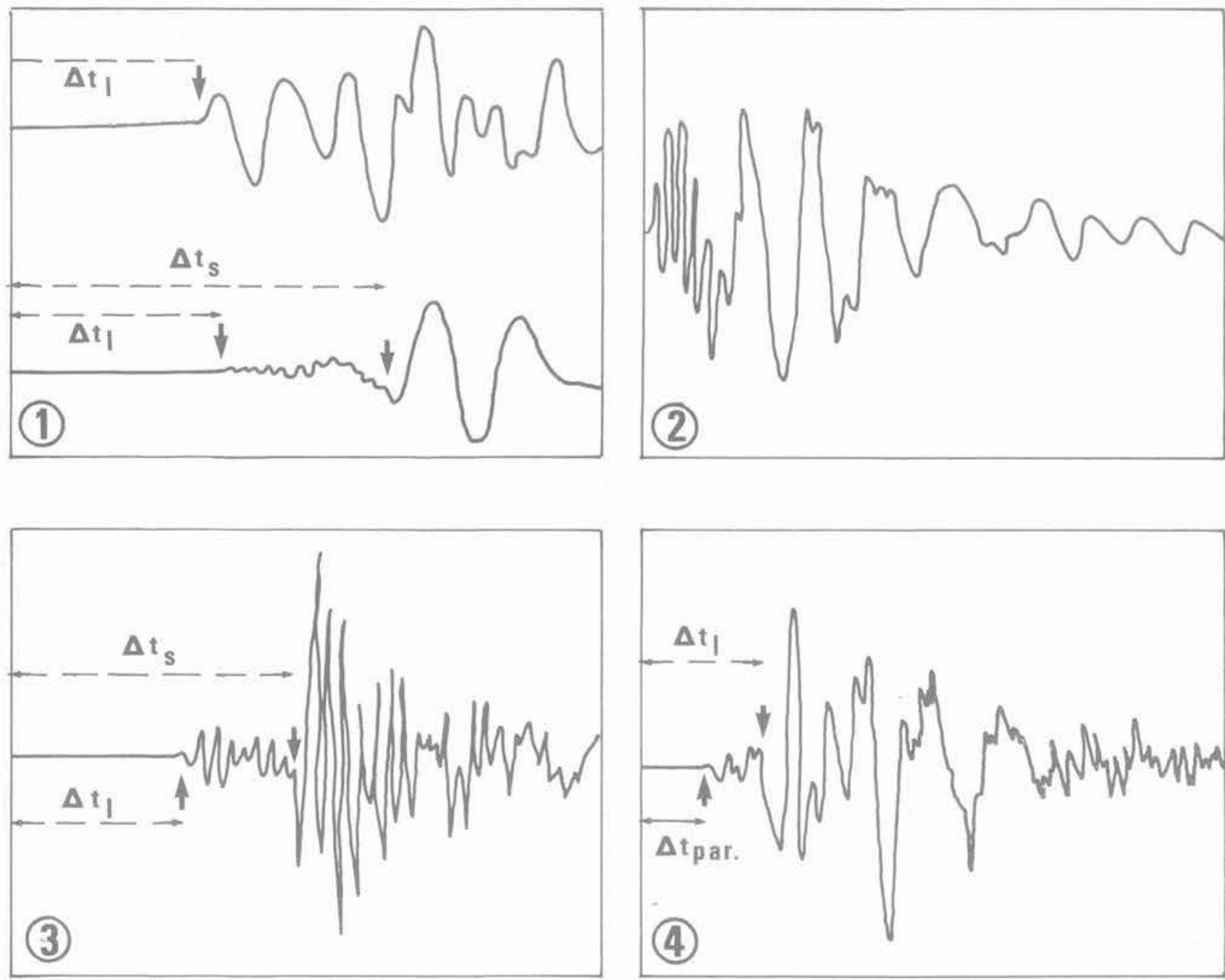

Fig. 3. - Signaux acoustiques enregistrés à l'œedomètre.

1. Signaux reçus dans du Texsol-Fontainebleau sec par un capteur équipé de céramiques "longitudinales»" et " transversales 》 superposées.

En haut : arrivée des ondes longitudinales.

En bas: arrivée des ondes transversales.

2. Signal reçu capteur contre capteur en ondes longitudinales.

3. Signal reçu dans du Texsol-Fontainebleau sec par un capteur équipé d'une céramique "transversale", les arrivées des ondes longitudinales (les plus rapides)

et des ondes transversales (plus lentes) se distinguent nettement.

4. Signal reçu dans du Texsol-Fontainebleau sec; devant l'arrivée normale des ondes longitudinales,

on distingue une arrivée parasite d'amplitude plus faible

due au passage des ondes par le corps de l'œdomètre. 
où $\mathrm{t}, \mathrm{T}$ et $\chi$ sont respectivement le temps de montée à la source, le temps de parcours et une constante proche de 0,5 .

- Ajustement avec une loi de comportement en comparant les mesures avec des résultats numériques.

- Décrément aléatoire en vibration libre.

\section{REPONSE VIBRATOIRE}

En laboratoire, les essais à la colonne résonnante sont les plus souvent utilisés pour évaluer le module dynamique en vibrations et le coefficient d'amortissement correspondant. Le principe consiste à appliquer une excitation harmonique à l'une des extrémités d'un échantillon cylindrique de sol et à enregistrer sa réponse (photo 1 ).

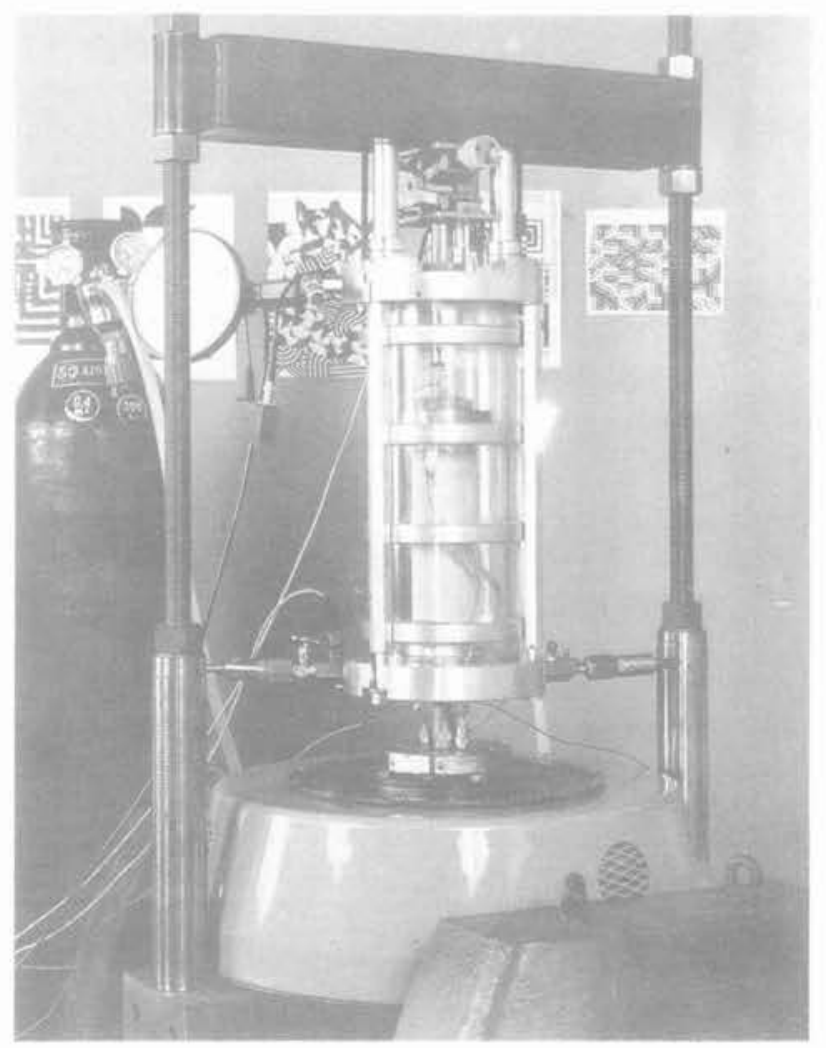

Photo 1. - Résonance longitudinale

à l'appareil triaxial cylindrique.

Il existe une grande diversité d'appareils de résonance dans le monde et en France (MECASOL, Laboratoire de Mécanique des Sols de l'École Centrale, Laboratoire de Mécanique des solides de l'École Polytechnique...). Les dispositifs expérimentaux et la méthode d'interprétation varient avec le type de conditions aux limites choisies. Les résultats conduisent cependant à des valeurs comparables des propriétés du sol testé. a) Vibrations longitudinales (MECASOL, École Centrale, Ecole Polytechnique).

En négligeant l'influence des forces d'inertie transversales dues à la contraction ou à la dilatation des sections droites, l'équation :

$$
E \frac{\partial^{2} \xi}{\partial x^{2}}=\rho \frac{\partial^{2} \xi}{\partial t^{2}}
$$

définit une propagation par ondes de célérité

$$
V_{L}=\sqrt{\frac{E}{\rho}}
$$

Cette célérité est un peu plus petite que celle des ondes longitudinales dans un solide à trois dimensions. Cette différence est due au fait que dans le cas de la tige, les déplacements latéraux sont possibles alors que dans le milieu à trois dimensions, il n'y a pas de déplacements latéraux.

b) Vibrations de torsion (MECASOL, École Polytechnique).

Dans le cas d'une section circulaire, l'équation du mouvement s'écrit :

$$
\mu \frac{\partial^{2} \theta}{\partial \mathrm{x}^{2}}=\rho \frac{\partial^{2} \theta}{\partial \mathrm{t}^{2}}
$$

Les équations de mouvement longitudinal ou de torsion peuvent être intégrées par deux procédés :

- l'un (d'ALEMBERT-EULER) met en évidence la propagation du mouvement,

- l'autre (BERNOULLI - développement en série de FOURIER) décrit l'aspect vibratoire ou périodique dans le temps comme une conséquence de la réflexion des ondes aux extrémités.

Les solutions élémentaires utilisées dans la solution de BERNOULLI sont des vibrations sinusoïdales stationnaires dont les fréquences sont déterminées par les conditions à la limite. En effet pour un système dont la position dépend d'un nombre fini $r$ de paramètres et lorsque les forces ne dépendent que de la position, n'importe quel petit mouvement peut s'obtenir en superposant $\mathrm{r}$ oscillations principales. La période et la phase sont les mêmes pour tous les points du système. Ces propriétés subsistent pour un milieu continu élastique avec une infinité de paramètres, les actions élastiques jouant le rôle des forces de rappel ne dépendant que de la position.

Au Laboratoire de Mécanique des Solides, on s'est attaché à obtenir une homogénéité de déformation en torsion comme en compression pour toute l'éprouvette en utilisant dans les essais de résonance une masse active $\mathrm{M}$ importante par rapport à la masse $\mathrm{m}$ du matériau testé $(\mathrm{M} / \mathrm{m}>3)$. Le déplacement et la déformation calculés le long de l'éprouvette dans le cas de la compression sont donnés dans la figure 5 . Les photos $2 \mathrm{a}$ et $2 \mathrm{~b}$ montrent l'homogénéité de dissipation thermique de l'éprouvette soumise à des sollicitations crois- 


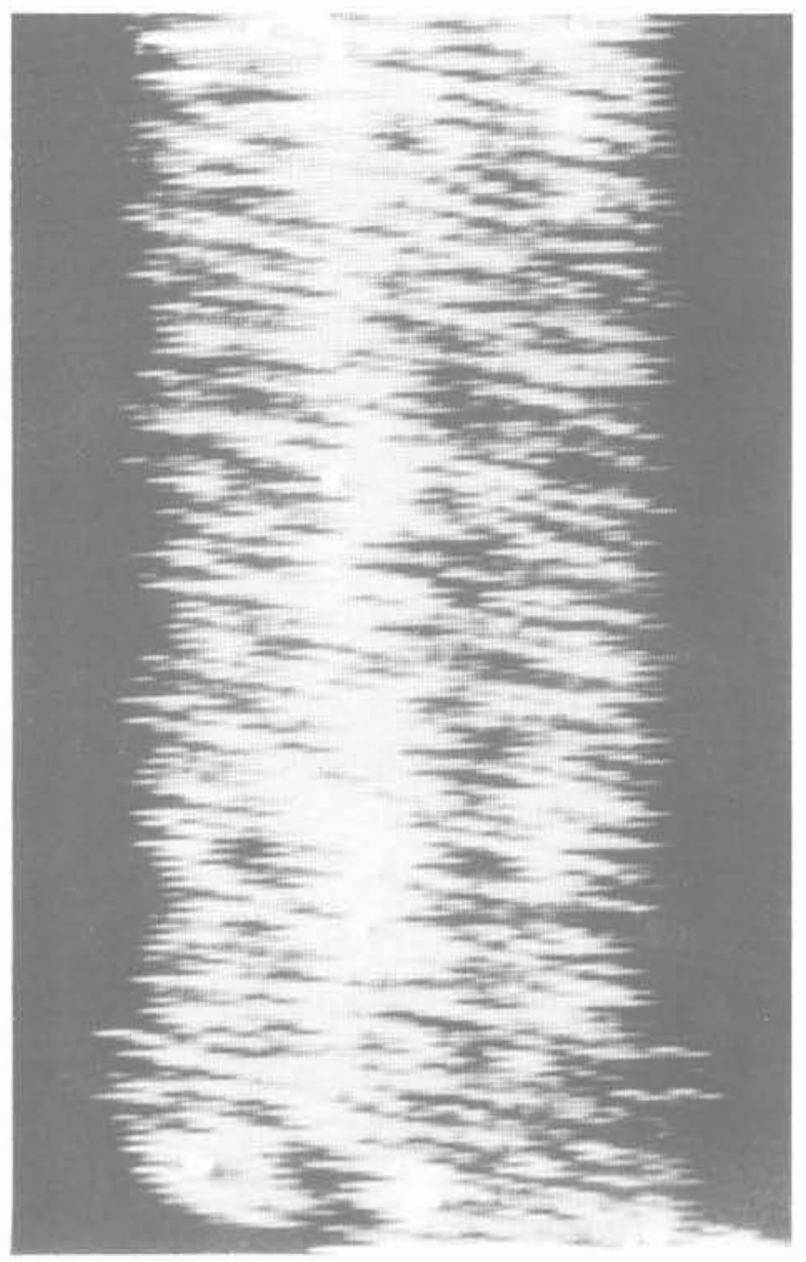

a) faible déformation $\varepsilon \sim 10^{-4}$

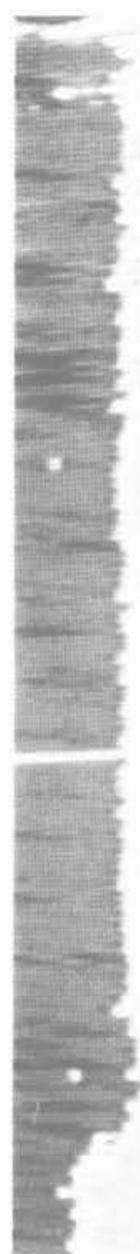

\section{b) déformation plus importante $\varepsilon \sim 10^{-3}$}

Photo 2. - Thermographie infrarouge d'un échantillon de sable en vibrations longitudinales: la masse active $M$ est trois fois plus importante que la masse $m$ de l'éprouvette.

On observe une uniformité de l'échauffement le long de l'échantillon.

santes. La photo 3 montre un ventre de déformation localisée lorsque la masse active $\mathrm{M}$ est faible $(\mathrm{M}=\mathrm{m})$.

Nous disposons d'autre part d'un système de résonance utilisant des échantillons de grand diamètre (300 mm) pour des mațériaux plus grossiers comme les graves.

Les figures $6 \mathrm{a}$ et $6 \mathrm{~b}$ présentent le module d'YOUNG E et le coefficient d'amortissement $D$ déterminés au triaxial cylindrique dynamique par un balayage en fréquence de $300 \mathrm{~Hz}$ à $10 \mathrm{~Hz}$ pour différentes pressions de confinement.

Tous les matériaux présentent une hystérésis mécanique sous déformation oscillatoire.

L'amortissement visqueux est défini pour les systèmes linéaires à multiples degrés de liberté (MDOF) ou simplement continu en multipliant les termes de vitesse par des coefficients constants. Ce type d'équation est utilisé également avec une excitation transitoire simple ou aléatoire.

L'amortissement hystérétique ou structural, aussi appelé à module complexe, semble donner un meilleur accord avec les réponses mesurées sur des structures à basse fréquence.

A amortissement relativement faible, le mouvement est contrôlé par la masse et la raideur, de sorte qu'il est possible de calculer un amortissement linéaire équivalent constant. Cette technique a été étendue aux systèmes à multiples degrés de libertés (MDOF) et continu avec un coefficient d'amortissement fonction de la fréquence afin d'ajuster les valeurs mesurées. Cette méthode n'est valable que si les forces d'amortissement sont faibles par rapport aux forces élastiques et d'inertie. 

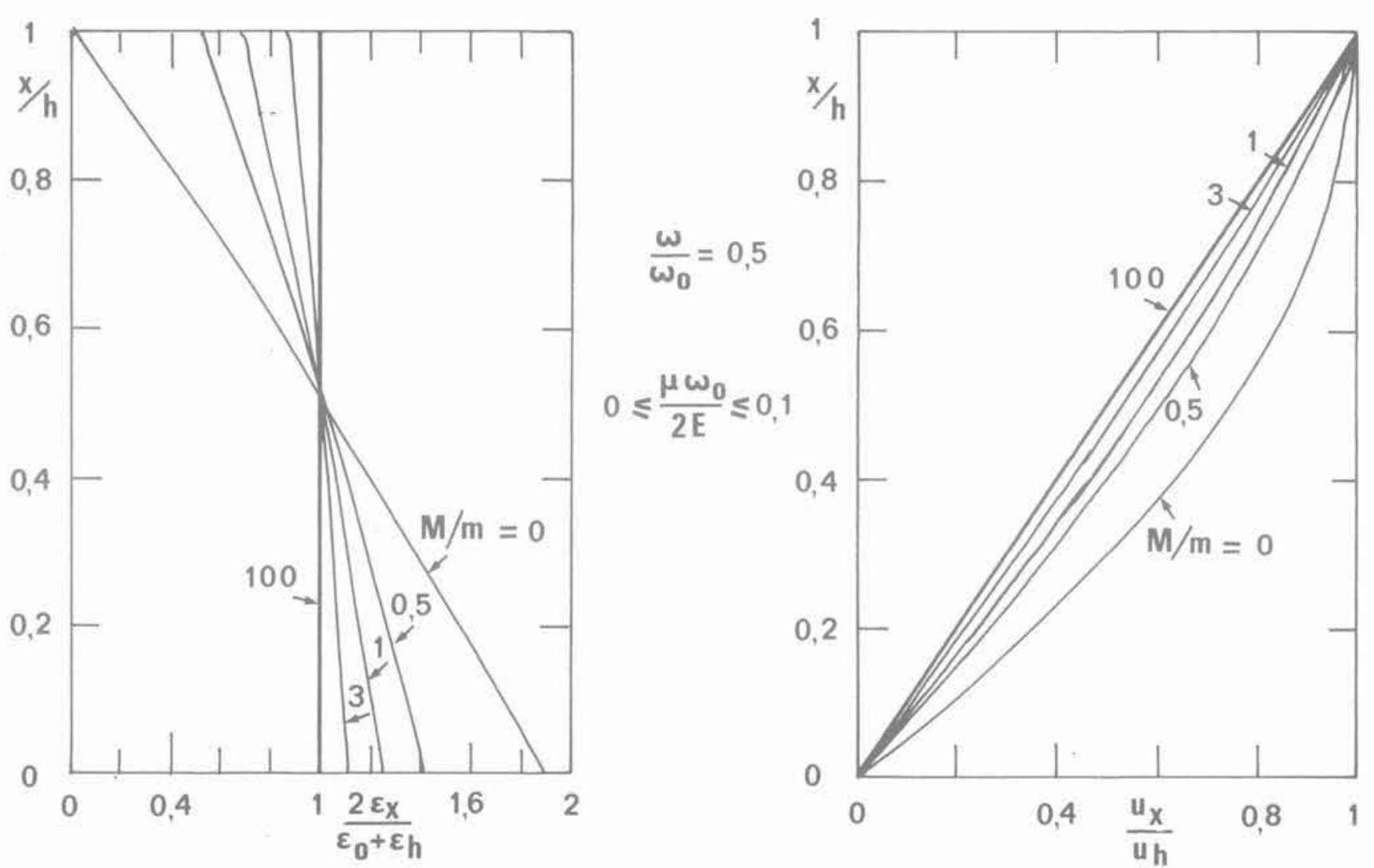

Fig. 5. - Déplacement et déformation en fonction de la masse active $M$ par rapport à la masse $m$ de l'échantillon.
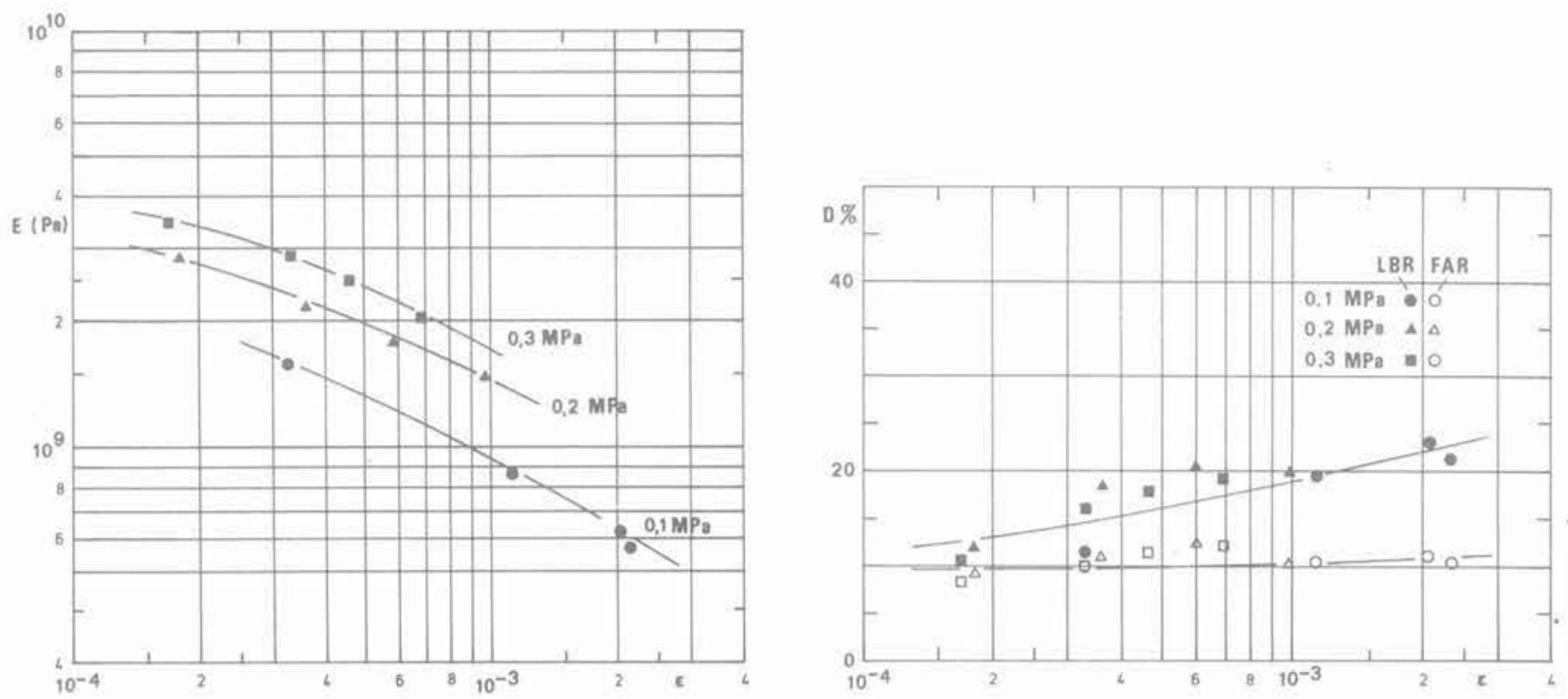

Fig. 6a. - Module longitudinal E d'une argile champenoise déterminé par des essais de résonance longitudinale à l'appareil triaxial cylindrique sous différentes pressions de confinement.

Fig. 6b. - Coefficient d'amortissement $D$ déterminé par la largeur de bande de résonance $L B R$ et par le facteur d'amplification à la résonance FAR de l'argile champenoise. 


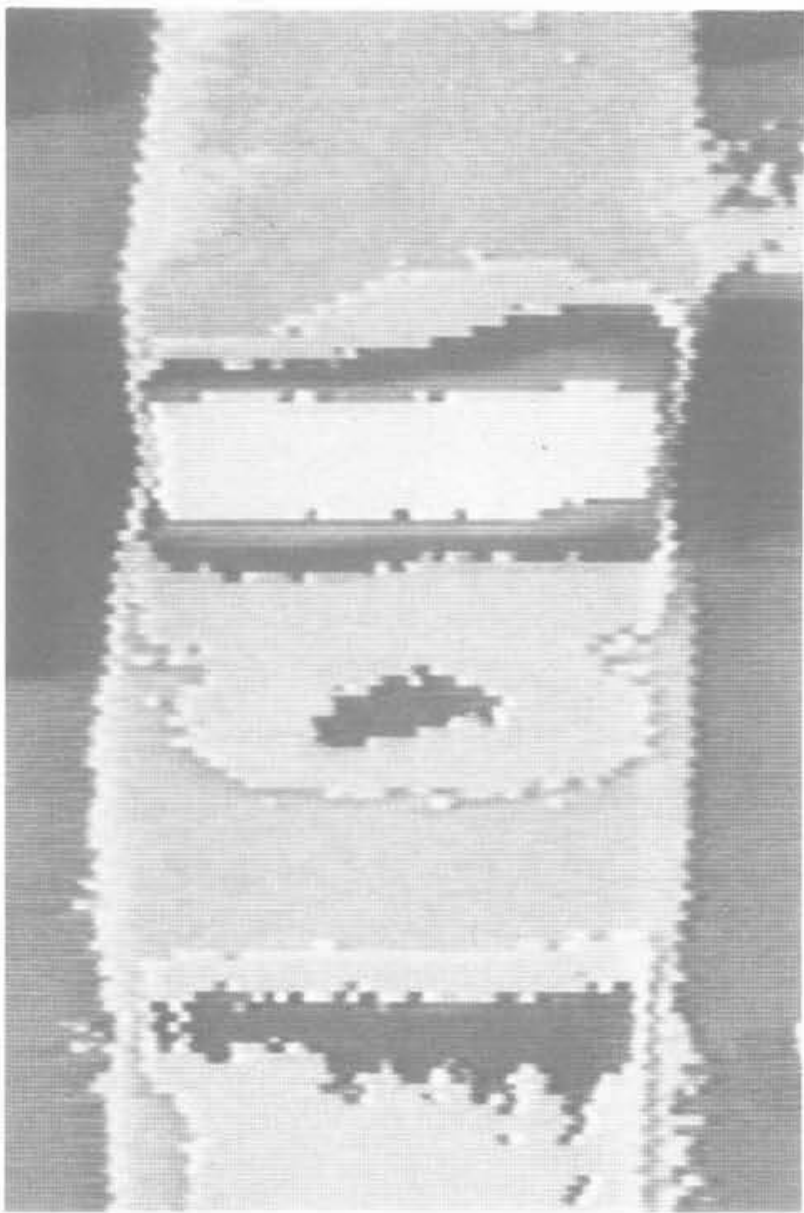

Photo 3. - Thermographie infrarouge d'un échantillon de sable en vibrations longitudinales .

la masse active $M$ est égale à la masse $m$ du spécimen.

On constate un échauffement important dans la zone de striction où les déformations sont plus importantes que dans les autres parties.

Le coefficient d'amortissement D peut être évalué par les différentes techniques suivantes:

A. Énergie emmagasinée ou dissipée par cycle de contrainte

$\mathrm{U}$ énergie de déformation élastique par unité de volume

D énergie d'amortissement par unité de volume

B. Module complexe

$$
\begin{aligned}
& E^{*}=E^{\prime}(1+i \eta)=\left|E^{*}\right| e^{i \phi} \\
& \left|E^{*}\right|=\left[E^{\prime 2}+E^{\prime \prime}\right]^{1 / 2}
\end{aligned}
$$

Module emmagasiné ou réel ou élastique

$$
\mathrm{E}^{\prime} \quad=\left|\mathrm{E}^{*}\right| \cos \varphi
$$

Module de perte ou de dissipation

$$
E^{\prime \prime}=\left|E^{*}\right| \sin \varphi
$$

C. Énergie relative

Coefficient de perte ou facteur d'amortissement

$$
\eta=\frac{D}{2 \pi U}=\frac{E^{\prime \prime}}{E^{\prime}}=2 D
$$

Facteur de qualité

$$
\mathrm{Q}=\frac{2 \pi \mathrm{U}}{\mathrm{D}}=\frac{1}{\eta}=\frac{1}{2 \mathrm{D}}
$$

D. Décroissance temporelle des vibrations libres décrément logarithmique $\delta=\frac{D}{2 U}$ constante de décroissance.

E. Atténuation spatiale des ondes atténuation logarithmique constante d'atténuation.

In situ, l'onde de RAYLEIGH offre la possibilité de déterminer le module de cisaillement. Les ondes stationnaires générées par un vibreur harmonique sont mesurées par des géophones qui détectent les points en phase avec le signal excitateur. La longueur d'onde ainsi estimée donne la vitesse des ondes de cisaillement (RICHART et al., 1970).

$\mathrm{V}_{\mathrm{R}}=\lambda_{\mathrm{R}} \mathrm{N}_{e}$ où $\mathrm{N}_{e}$ est la fréquence excitatrice.

Lorsque le profil géotechnique présente une couche de faible vitesse de propagation recouvrant une couche de forte vitesse, les ondes stationnaires de LOVE peuvent être utilisées pour évaluer les célérités des ondes de cisaillement (JONES, 1958).

De même, l'utilisation d'une excitation stationnaire en forage a permis de mesurer correctement les paramètres du sol en faibles déformations (BALLARD, 1976). Le calcul des vitesses des ondes de cisaillement et des déformations de cisaillement s'effectue avec la mesure des différences de phase entre la source et les capteurs placés dans des trous de forage adjacents.

La réponse vibratoire des fondations en oscillation verticale, horizontale, en rotation ou en torsion a également été utilisée pour évaluer le module de cisaillement et le coefficient d'amortissement aussi bien in situ qu'en centrifugeuse (LEGUAY et al., 1984).

L'amélioration constante des techniques de mesures (capteurs de plus en plus performants), de l'électronique et des analyseurs digitaux a permis l'utilisation de l'analyse modale pour déterminer les propriétés dynamiques du sol. En effet le test modal est un processus de construction d'un modèle mathématique pour décrire les propriétés vibratoires d'une structure, basé sur les données expérimentales. Il permet donc d'estimer avec précision les fréquences naturelles et les niveaux d'amortissement modal grâce à des procédures d'ajustement pour arriver à la plus proche description du comportement réellement observé. 
Certaines propriétés géométriques des courbes de fonction de réponse en fréquence (FRF) du système à un seul degré de liberté (SDOF) ou oscillateur, peuvent aider la distinction entre amortissement visqueux et amortissement hystérétique.

Ainsi à faible amortissement $(<1 \%)$, la mobilité présente une pseudosymétrie par rapport à la verticale de la résonance dans le plan de BODE. Dans le diagramme de NYQUIST, la mobilité d'un système à amortissement visqueux et la réceptance d'un système à amortissement hystérétique apparaissent exactement sous forme de cercle lorsque la fréquence est balayée de 0 à $\infty$.

En effet, lorsque l'amortissement est visqueux, l'équation du mouvement en vibration forcée s'écrit :

$$
\mathrm{m} \ddot{\mathrm{x}}+\mathrm{dx}+\mathrm{kx}=\mathrm{Fe} \mathrm{e}^{\mathrm{j \omega t}}
$$

Avec la solution $\mathrm{x}^{(t)}=\mathrm{xe}^{\mathrm{t} \text { tot}}$, la réceptance est de la forme :

$$
\alpha(\omega)=\frac{1}{\left(k-\omega^{2} m\right)+i \omega d}
$$

La mobilité est alors donnée par :

$$
\begin{aligned}
Y(\omega) & =i \omega \alpha(\omega)=\frac{i \omega}{\left(k-\omega^{2} m\right)+i \omega d} \\
& =\frac{\omega^{2} d+i \omega\left(k-\omega^{2} m\right)}{\left(k-\omega^{2} m\right)^{2}+(\omega d)^{2}}
\end{aligned}
$$

En posant

$$
\begin{aligned}
& U=\operatorname{Re}(Y)-\frac{1}{2 d} \\
& V=\operatorname{Im}(Y)
\end{aligned}
$$

on constate que $\mathrm{U}^{2}+\mathrm{V}^{2}=\left(\frac{1}{2 \mathrm{~d}}\right)^{2}$

La mobilité est donc représentée dans le diagramme de NYQUIST par un cercle de rayon $\frac{1}{2 \mathrm{~d}}$ et centré sur l'axe réel.

Dans le cas de l'amortissement hystérétique, la réceptance est donnée par :

$$
\alpha(\omega)=\frac{1}{\left(k-\omega^{2} m\right)+i h}=\frac{\left(k-\omega^{2} m\right)-i h}{\left(k-\omega^{2} m\right)^{2}+h^{2}}
$$

On peut voir que :

$$
(\operatorname{Re}[\alpha(\omega)])^{2}+\left(\operatorname{Im}[\alpha(\omega)]+\frac{1}{2 h}\right)^{2}=\left(\frac{1}{2 h}\right)^{2}
$$

La réceptance d'un système amorti hystérétiquement dessine donc un cercle de rayon $\left(\frac{1}{2 h}\right)$ et centré sur laxe imaginaire dans le diagramme de NYQUIST.

\section{COMPORTEMENT CYCLIQUE}

Sous sollicitations cycliques, le matériau sol se comporte comme un assemblage de particules solides baignées ou non dans l'eau interstitielle. Les déformations proviennent alors essentiellement de la modification de la structure granulaire, c'est-à-dire des réarrangements de la fabrique avec changement du nombre des contacts intergranulaires.

Les principaux mécanismes de déformation possibles sont un serrage et surtout un déplacement relatif des grains pouvant être associés à une déformation volumique très significative, en particulier lors d'une phase de dilatance. Les glissements des grains deviennent alors suffisamment importants pour provoquer un désenchevêtrement rapide de la structure granulaire.

L'étude expérimentale dans le cadre du concept de l'état caractéristique ou état de dilatance nulle a permis de reconnaître les différents comportements cycliques du sol sableux à l'appareil triaxial cylindrique : adaptation, accommodation, rochet en condition drainée ou à sec et durcissement cyclique, amollissement cyclique, liquéfaction en condition non drainée (fig. 7). En fonction du niveau déviatorique des contraintes $\eta=q / p$ et du trajet de chargement suivi, on peut estimer et mesurer les principaux paramètres de déformation et d'amortissement en fonction du nombre de cycles de chargement. Les caractéristiques de liquéfaction sont souvent mesurées lors des essais à déformation axiale contrôlée.

Lorsqu'on essaie de reproduire les conditions dynamiques in situ, l'essai triaxial cyclique présente quelques limitations :

- Les mesures des déformations inférieures à 10-2 sont très difficiles et ne sont pas précises.

- Les phases d'extension et de compression à chaque cycle produisent des résultats différents à cause de la rotation des axes de contrainte : les boucles d'hystérésis ne sont pas symétriques en déformation contrôlée et les éprouvettes sont souvent sujettes à des strictions en contrainte contrôlée.

- L'indice des vides se redistribue à l'intérieur de l'échantillon.

- Le frettage des extrémités peut être significatif dans certains cas.

Pour le calcul sismique, les techniques utilisant le cisaillement simple et de torsion cycliques paraissent plus appropriées malgré les difficultés aussi bien de principe que de réalisation d'un état parfait de cission du sol.

Les caractéristiques du comportement cyclique en place peuvent être obtenues à l'aide des essais pressiométriques cycliques et des chargements cycliques des pieux. Parfois les techniques de chargement cyclique de plaque et de cission cyclique en forage ont été également utilisées.

Le principe consiste à appliquer des contraintes (ou déformations) sur une surface donnée et à enregistrer les déplacements engendrés. On obtient ainsi les relations contraintes-déformations, les variations de 


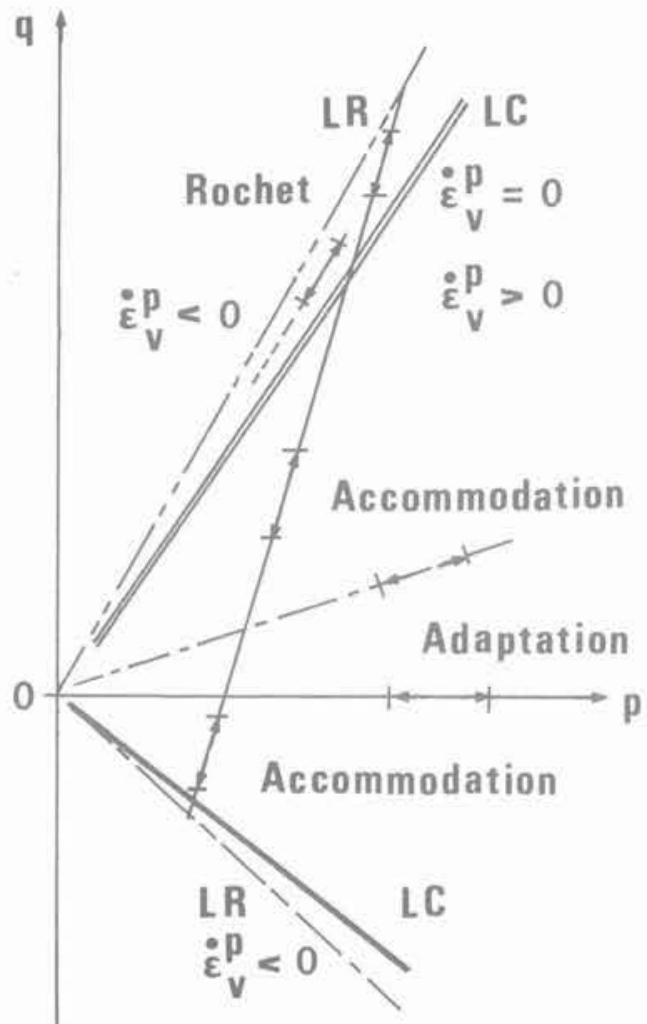

EN CONDITION DRAINEE

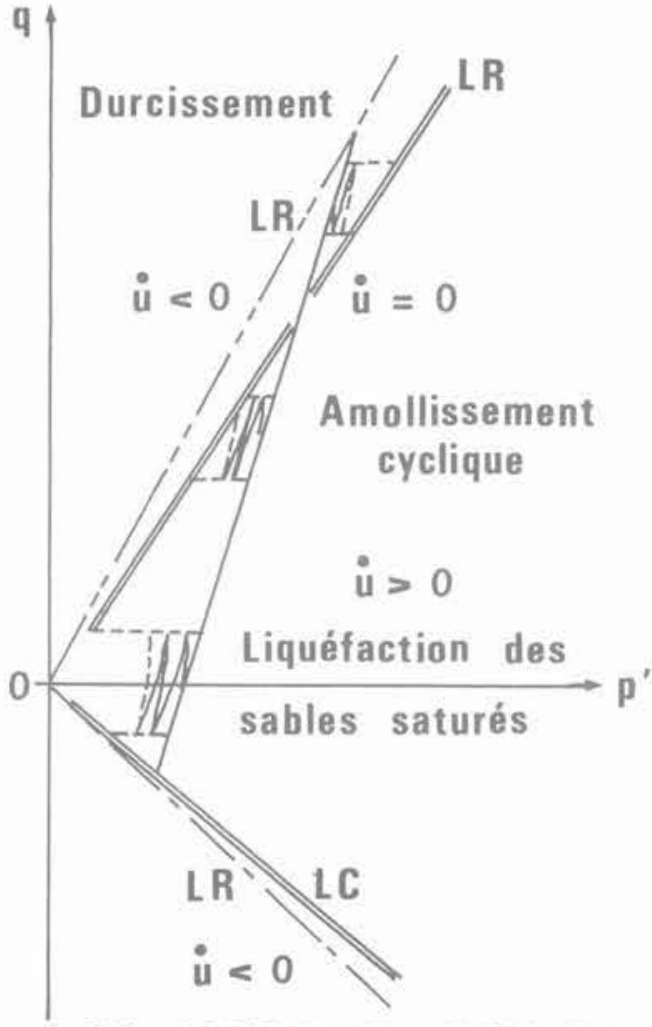

EN CONDITION NON DRAINEE

Fig. 7. - Divers comportements cycliques facilement observables à l'appareil triaxial conventionnel.

volume, la résistance limite du sol et éventuellement le développement de la pression interstitielle en milieu saturée avec le piézocone ou le pénétromètre SPT. L'amortissement est déduit de la dissipation d'énergie à partir de la boucle du chargement cyclique enregistrée.

\section{CHOCS ET EXPLOSIONS :}

\section{EFFET DU TAUX DE DÉFORMATION}

En laboratoire, de nombreux essais dynamiques des sols utilisant l'appareil triaxial cylindrique, de torsion, de cisaillement direct ont été conduits pour déterminer la différence entre les modules de déformation en statique et en dynamique, ainsi que les résistances à la rupture (CASAGRANDE et SHANNON, 1948). Un facteur taux de déformation (strain rate factor) caractérise l'effet de la vitesse de sollicitation. La figure 8 montre la tenue d'un échantillon de Texsol-Fontainebleau après un choc développant 19,62 J produit par une masse tombant sur sa tête.

In situ, l'Université de Zurich a développé dans les années 60 un canon à eau capable de déterminer la raideur dynamique du sol de support (BAMERT et al., 1967).

Fig. 8. - Tenue au choc d'un échantillon de Texsol-Fontainebleau.
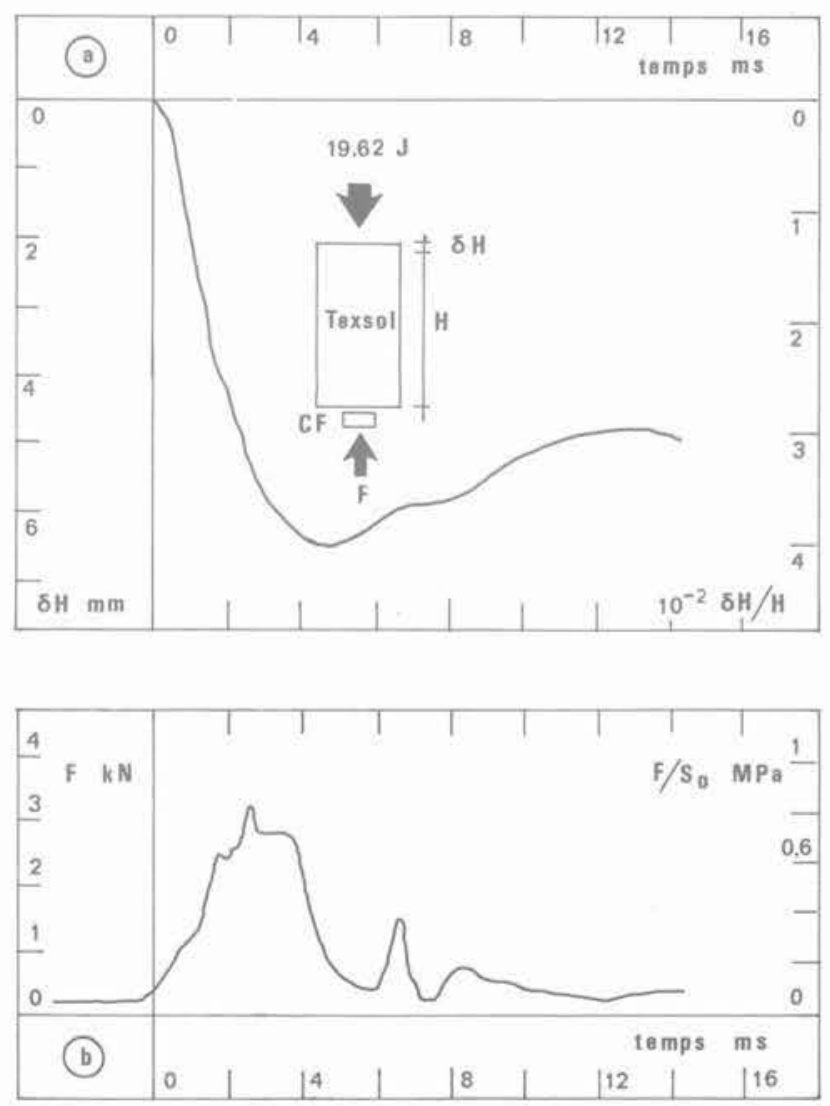
Pour étudier le comportement du sol lors des explosions thermonucléaires, l'essai CIST (cylindrical in situ Test) de l'armée américaine consiste à faire exploser une charge répartie d'explosif PETN dans une cavité cylindrique tubée. L'explosion créa des pressions de Pordre de $42 \mathrm{MPa}$ et causa la rupture des matériaux situés en champ proche. Les paramètres élastoplastiques du sol ont été déduits par ajustement des courbes de calcul avec celles mesurées in situ (BRATTON et HIGGINS, 1978). D'autres essais analogues ont été également réalisés : PM (PLANAR METHOD) et SISM (SPHERICAL IN SITU METHOD).

SHANNON et WILSON en collaboration avec AGBABIAN Associates ont développé l'essai d'impulsion intitulé "SW-AA " pour évaluer in situ les modules non linéaires et les coefficients d'amortissement du sol en grandes déformations couvrant la gamme $10^{-5}$ à $0,5 \cdot 10^{-2}$. L'excitation est produite par un marteau tombant sur la tête d'un système d'ancrages cylindriques. Les modules de déformation sont déduits des vitesses de propagation des ondes et le coefficient d'amortissement a été évalué par l'aire de la boucle d'hystérésis.

\section{CONCLUSION}

Des techniques fiables de mesure des propriétés dynamiques des sols sont disponibles aussi bien en laboratoire qu'in situ.

Cependant les résultats obtenus comparés sont rarement concordants parce que le principe de mesure, la qualité des échantillons et les conditions aux limites ne sont pas analysés correctement dans les deux cas.

La prise en compte du sol dans les calculs de réponse dynamique s'effectue à travers une modélisation du sol. Il est donc plus logique de déterminer les paramètres, aussi bien en laboratoire qu'in situ, à l'aide des techniques appropriées ou compatibles avec cette modélisation. L'analyse modale en laboratoire et en place s'est révélée très prometteuse pour les prochaines années.

\section{BIBLIOGRAPHIE}

BALLARD R.F. (1976), Method for cross-hole seismic testing, ASCE, J.G.E.D., vol. 102, GT 12, déc. 1976, pp. 1261-1273.

BAMERT E., SCHNITTER G. and WEBER M. (1967), A Field Method of Determining Soil Properties by Impact Loading, Proceedings of International Symposium on Wave Propagation and Dynamic Pro- perties of Earth Materials, Albuquerque, N.M., Aug. 23-25, pp. 265-274.

BARKAN D.D. (1962), Dynamics of Basis and Foundations, Mc Graw-Hill Book Company.

BRATTON J.L. and HIGGINGS C.J. (1978), Measuring Dynamic In Situ Geotechnical Properties, Earthquake Engineering and Soil Dynamics, Geotechnical Engineering Division ASCE Specialty Conference Pasadena, June 19-21, pp. 272-289, vol. I.

CASAGRANDE A. and SHANNON W.L. (1948), Stress Deformation and Strength Characteristics of Soils under Dynamic Loads, Proceedings of the Second International Conference on Soil Mechanics and Foundations Engineering, Rotterdam, vol. V, pp. 29-34.

EWINS D.J. (1984), Modal Testing: Theory and Practice, Research Studies Press Ltd, John Wiley \& Sons Inc.

HADJ HAMOU A. (1983), Contribution à l'étude du comportement des Sols pulvérulents sous chargements cycliques et dynamiques, Thèse de Docteur Ingénieur, E.N.P.C., Paris, décembre 1983.

HAGHGOU M. (1985), Mesure in situ de l'amortissement interne des Sols, Sol Engineering, Études géotechniques.

HARDIN B.O. (1978), The Nature of Stress-Strain Behavior for Soils, Earthquake Engineering and Soil Dynamics, vol. I, ASCE 1978 , pp. 3-90.

HARRIS C.M. and CREDE C.E. (1976), Shock and Vibration Handbook, 2nd ed. Mc Graw-Hill Book Company.

JONES R. (1958), In situ Measurement of the Dynamic Properties of Soil by vibration Methods, Géotechnique, vol. VIII, $n^{\circ} 1$, Mars, pp. 1-21.

LEGUAY P., MORLIER P. et RIVIERE J. (1984), Essais dynamiques de fondations en centrifugeuse, Revue Française de Géotechnique, $n^{\circ} 28$, $1^{\text {er }}$ trim. 1984.

LUONG M.P. (1986), Characteristic Threshold and Infrared Vibrothermography of Sand, ASTM Geotechnical Testing Journal, vol. IX, n², June 1986 U.S.A. MANDEL J. (1966), Cours de Mécanique des Milieux Continus, tome II, Mécanique des Solides, chap. VIII et annexe XIX, Gauthier Villars.

MINDLIN R.D. and DERESIEWICZ H. (1953), Elastic Spheres in Contact Under Varying Oblique Forces, Journal of Applied Mechanics, vol. XX, 1953, pp. 327-344,

NAUROY J.F. (1975), Étude de la vitesse de propagation des ondes acoustiques dans les sédiments argilosableux à différents stades de leur compaction, Thèse Docteur Ingénieur, Paris-VI.

RICHART F.E. Jr, HALL J.R. Jr, and WOODS R.D. (1970), Vibrations of Soils and Foundations, Prentice Hall, Englewood Cliffs, N.J., 414 pages.

WOODS R. (1978), Measurement of Dynamic Soil Properties, Earthquake Engineering and Soil Dynamics, Specialty Conference Pasadena June 15-21, Geotechnical Engineering Division ASCE 1978, vol. I, pp. 91-178. 